\title{
The effect of visibility on eye-movement parameters in reading
}

\author{
J. KEVIN O'REGAN, ARIANE LÉVY-SCHOEN, and ARTHUR M. JACOBS \\ CNRS, Paris, France
}

\begin{abstract}
The effect of visibility on eye-movement parameters was investigated. As a measure of visibility, the notion of "visual span" was introduced. A first experiment, in which a simple letterrecognition task was used, directly measured the changes in visual span produced by changing viewing distance and character spacing. The results of this experiment were used as a reference for a second experiment in which the same visibility changes were made, but in which subjects read short texts while their eye movements were monitored. Saccade sizes were affected not primarily by visual span, but by other factors, possibly related to word boundary detection or linguistic processing. Fixation durations appeared to be strongly affected by the proximity of the letters to the subject's acuity threshold.
\end{abstract}

The purpose of the present study was to investigate the effect of visibility changes on eye-movement behavior in reading. Visibility was studied in relation to eye movements by Hackmann and Tinker (1947), Paterson and Tinker (1947), and Tinker and Paterson (1955), but these authors' purpose was to find the optimal conditions for reading, and not the reason why these conditions were optimal.

A measure of "visibility" can be obtained by determining the probability of recognizing letters in the fovea. But this measure may not be relevant to understanding reading behavior, because it neglects the effect of parafoveal and peripheral vision. "Visual span," that is, the size of the region around the eye's fixation point in which letters can be recognized with a given accuracy, would appear to be more relevant in determining saccade size and fixation durations in reading. In adjusting to poor visibility conditions, in which visual span is small, we expect the eye to make smaller saccades. In good visibility conditions, in which the span is large, we expect large saccades. The situation for fixation durations is harder to predict. On the one hand, when span is small, there is less to process and fixations should be short. On the other hand, the individual letters are harder to see, so durations should be long. The present study investigated these possibilities.

The notion of "span" has been much used in the eye-movement literature, (see, e.g., discussions by McConkie, 1983, and Rayner, 1983), and it is important to note that the definition we are using here is peculiar. Here, when we refer to "visual" span, we restrict ourselves to a measure that reflects only the initial, largely sensory stages of processing, and which is

The authors' mailing address is: Groupe Regard, Laboratoire de Psychologie Expérimentale, Université René Descartes, EPHE, CNRS, 28 rue Serpente, 75006 Paris, France. not influenced by linguistic context. It is clear that if span is measured in conditions in which contextual information is being used to help in letter recognition, then the span will be bigger than when there is no context: the number of letters that can be reported when the eye fixates in a string will be much larger if the string is a word. In previous work (O'Regan, 1979), we have called this larger span the "perceptual" span, to distinguish it from the purely "visual" span to be considered here.

Changes in visual span can be obtained in a variety of ways. In the present experiments, we used two methods which we had studied from a theoretical standpoint (cf. O'Regan, 1983): changing viewing distance and changing letter spacing. The visual-span changes measured when these parameters are manipulated in a simple character-recognition task (visibility experiment) are then used as reference values in interpreting eye-movement behavior in a second task (reading experiment), in which identical manipulations are performed in a situation in which subjects read short texts.

\section{VISIBILITY EXPERIMENT}

\section{Method}

In the present character-recognition task, we wished each chosen visibility condition to have a counterpart in the subsequent reading experiment. In reading, however, letters never appear alone, but are flanked by other letters. This is known to reduce their visibility significantly (cf. Bouma, 1973). In the present experiment, the same flanking of letters by other letters must therefore also be used. However, if target letters are flanked by other letters, then, first, to avoid memory load, a way must be found to indicate to the subject which letter is the target. Second, even if this is done, response competition between targets and nontargets can occur, and this would obscure the purely sensory effects. After pilot experiments using a variety of displays, these problems were solved by using random digits between 1 and 9, rather than letters, to flank the target letters. It was assumed that, as far as low-level visual 
processes were concerned, the masking effect of digits on letters would be about the same as that of letters on letters.

To reduce the number of trials needed to obtain reliable measures, two methods were used. First, a reduced alphabet consisting of 10 uppercase letters (A, C, E, K, L, P, S, T, X, and Y) was used. The particular subset used was chosen at random. It was assumed that the variation in visual span with eccentricity would follow the same laws as when the complete alphabet is used, albeit with a better report accuracy. Second, two targets were presented in each trial. These were symmetrically placed on either side of the fixation point, at a given eccentricity. This had the advantage of maintaining a symmetrical display in which there was less tendency for the subject to move his eyes. It was assumed that having to report two letters did not significantly affect memory load, and should, in any case, not interact with eccentricity.

Two methods were used to vary the conditions of visibility. They were chosen because a simple model (O'Regan, 1983) can, to some extent, describe their effect on visual span. The first method consisted of changing viewing distance. Four viewing distances-30, 60,90 , and $120 \mathrm{~cm}-$ ware used. The second method consisted of changing character spacings. In normal spacing, targets were presented with a masking digit flush on each side of it; in double spacing, exactly the same characters were used, but masking digits were now displayed one character space away from the target letter, as illustrated in the lines marked (a) in Figure 1.

Target letters could appear at seven eccentricities ranging from 4 to 11 character spaces from the fixation point. One character space measured $2.5 \mathrm{~mm}$. At $30,60,90$, and $120 \mathrm{~cm}$, this corresponds to $0.48,0.24,0.16$, and 0.12 deg of arc, respectively. So that subjects had no uncertainty about where in their visual field to expect target letters, trials were blocked by eccentricity. Within each constant eccentricity block, there were 20 trials, in which a pair of targets was presented. Within such a block, each of the 10 target letters occurred four times in random order, on one or the other side of the fixation point.

There were four subjects. To obviate effects due to practice or to
Table 1

\begin{tabular}{|c|c|c|c|c|c|c|c|c|}
\hline \multirow{2}{*}{$\begin{array}{l}\text { Sub- } \\
\text { ject }\end{array}$} & \multicolumn{8}{|c|}{ Order of Presentation } \\
\hline & 1 & 2 & 3 & 4 & 5 & 6 & 7 & 8 \\
\hline 1 & 30 & $\underline{90}$ & 60 & $\underline{120}$ & $120^{*}$ & $\underline{60}^{*}$ & $90^{*}$ & $\underline{30}^{*}$ \\
\hline 2 & 60 & 30 & 120 & $\overline{90}$ & $90^{*}$ & $1 \overline{20}^{*}$ & $30^{*}$ & $\overline{60} *$ \\
\hline 3 & $\overline{90} *$ & $120^{*}$ & $30 *$ & $60^{*}$ & 60 & 30 & 120 & 90 \\
\hline 4 & $120^{*}$ & $60^{*}$ & $90^{*}$ & $30^{*}$ & 30 & 90 & 60 & 120 \\
\hline
\end{tabular}

Note-The numbers within the table are the viewing distances in centimeters. Underlined numbers = normal spacing; not underlined numbers $=$ double spacing. $\quad$ Eccentricities decreasing, otherwise increasing.

associations between the distance, spacing, and eccentricity variables, the design was built in the following way. For the normalspace condition, a Latin square was constructed with 4 subjects $x$ 4 distances. A mirror image of this Latin square was constructed for the double-space condition, which had the opposite order of distances. The two Latin squares were interleaved so that subjects saw normal and double-space conditions in alternate order. The Latin squares were chosen so that the final order of distances was such that the distances never increased or decreased twice in succession. For half the subjects, the seven target-letter eccentricities within a condition were given in ascending order in the first four conditions and in descending order in the last four conditions. This was reversed for the other half of the subjects. The design is given in Table 1.

The experiment was controlled by an IMS computer. Stimuli appeared on an ADM 3A display terminal. This has a $9 \times 7$ character matrix. Lighting conditions were photopic and were kept identical across subjects, as were screen brightness and contrast. To begin each trial, the subject fixated a small gap between two fixation lines and pressed a button. A stimulus frame flashed up for $150 \mathrm{msec}$, short enough for no eye movement to occur. The subject

NORMAL SPACING

(a)

(b)
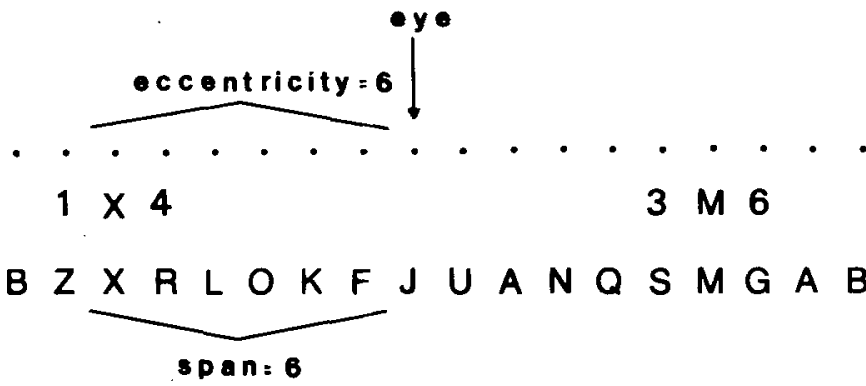

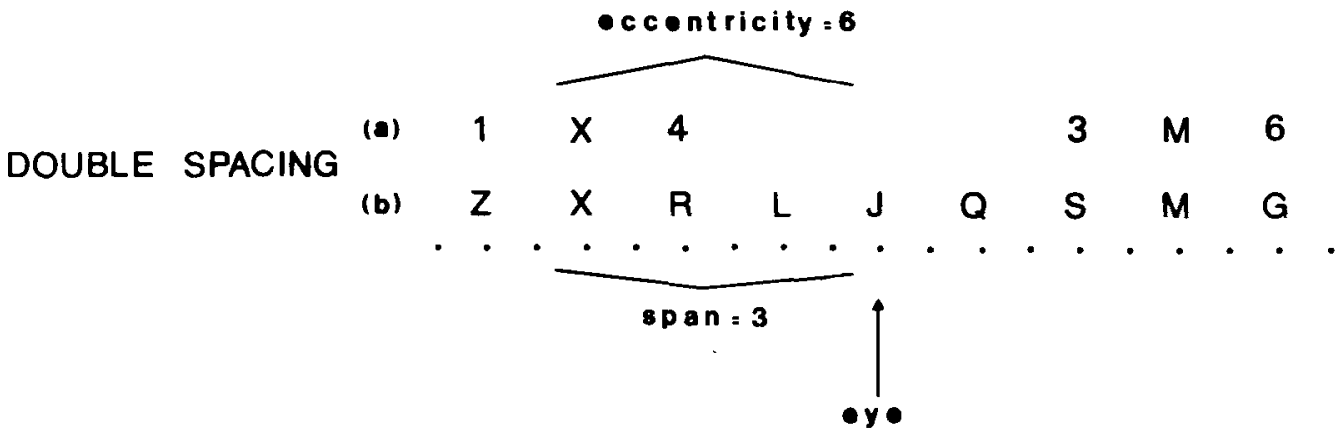

Figure 1. Lines marked (a) show examples of stimulus arrays used in the normal- and double-space conditions. The examples correspond to an eccentricity of 6 . In the double-space condition, this corresponds to a span, measured in letters, of 3 , since the letters are double spaced. This is illustrated in the lines marked (b). Characters used in the real display were generated in a $9 \times 7$ dot matrix by the ADM 3A terminal. 
indicated verbally the two target letters perceived, and these were noted by the experimenter. The subjects were practiced, having previously participated in many thousands of trials of pilot work.

\section{Results}

Figure 2 shows psychometric curves giving the probability of correct response as a function of ec- centricity, measured in character spaces. The curves were derived by taking the mean of the proportion of correct responses for the four subjects at each eccentricity ${ }^{1}$ and correcting for the chance response level, which was $1 / 10$ th, since there were 10 possible target letters. If $P$ is the proportion correct, the plotted value is $(P-0.1) /(1-0.1)$.

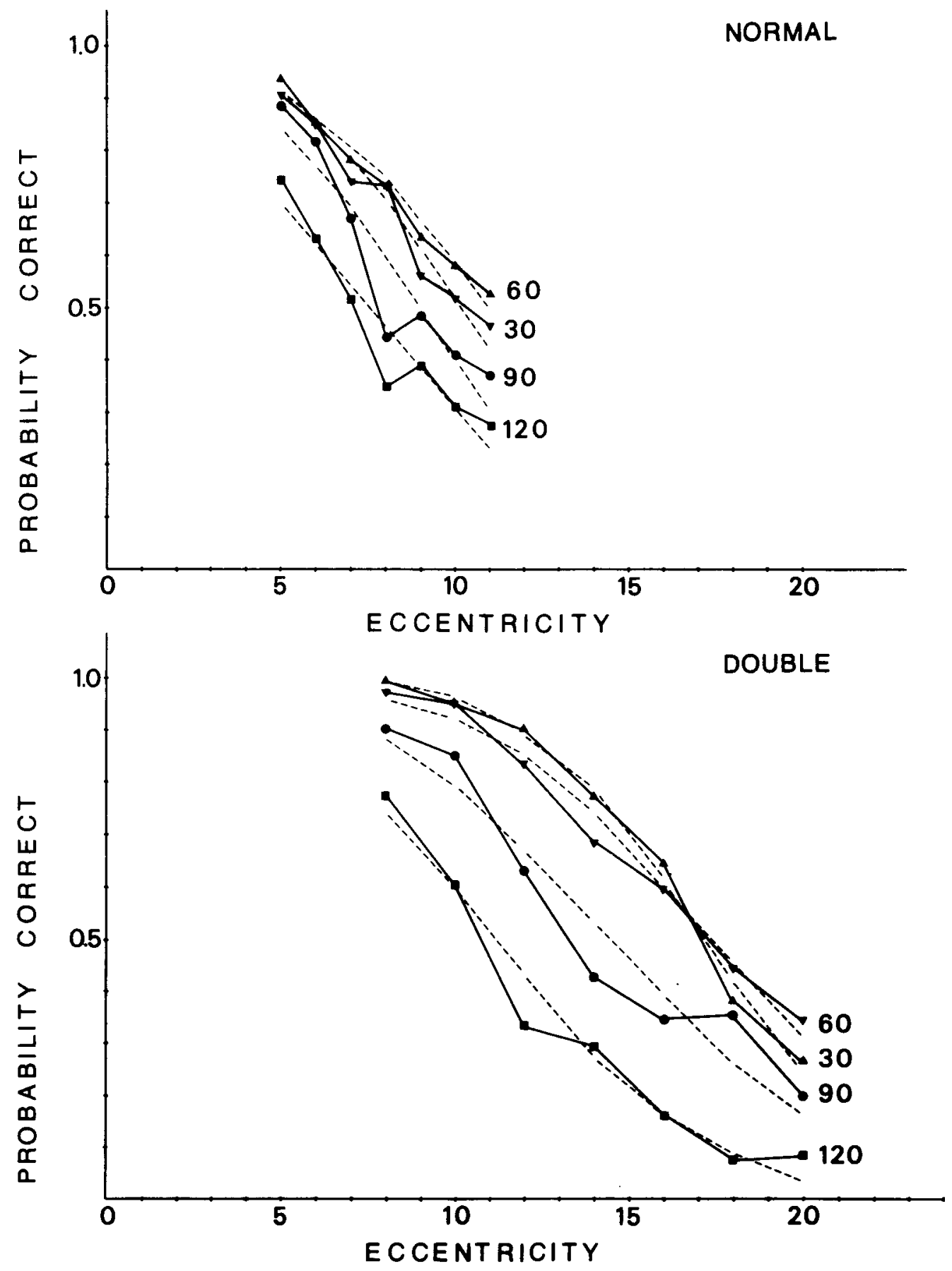

Figure 2. Means, across the four subjects, of the probability of correctly reporting a letter at a given eccentricity in the visibility experiment. Data are corrected for chance. Each curve corresponds to a given viewing distance, ranging from 30 to $120 \mathrm{~cm}$. The dotted lines show ogives fitted to the data using the probit method (Finney, 1947), 
Of interest in the reading experiment is visual span. This will be defined as the eccentricity within which letters can be seen with better than $50 \%$ probability of being correct. The choice of $50 \%$ is arbitrary. Assuming that the results of the visibility experiment apply to character strings, visual span can be derived from the psychometric curves by finding the eccentricity at which each curve crosses the $50 \%$ correct point. To determine the $50 \%$ points, a probit analysis (Finney, 1947) was done. The technique involves fitting the psychometric functions to the normal density ogive. The fitted curves are indicated as dotted lines in Figure 2. The eccentricities at the $50 \%$ point are plotted in Figure 3 . Note that, for compatibility with the reading experiment, the relevant measure of visual span is number of letters that can be processed on each side of the fixation point, not the eccentricity in number of character spaces of the farthest away letter that can be identified. While for the normalspace condition these two numbers are the same, for the double-space condition, number of letters visible is half the eccentricity in character spaces, since the letters are double spaced. This is explained in the lines marked (b) in Figure 1. Note also that, in the literature, "span" normally refers to the sum of the sizes of the zone on each side of the fixation point in which information is gathered. ${ }^{2}$ Here, for compatibility with the visibility measures, our "span" is

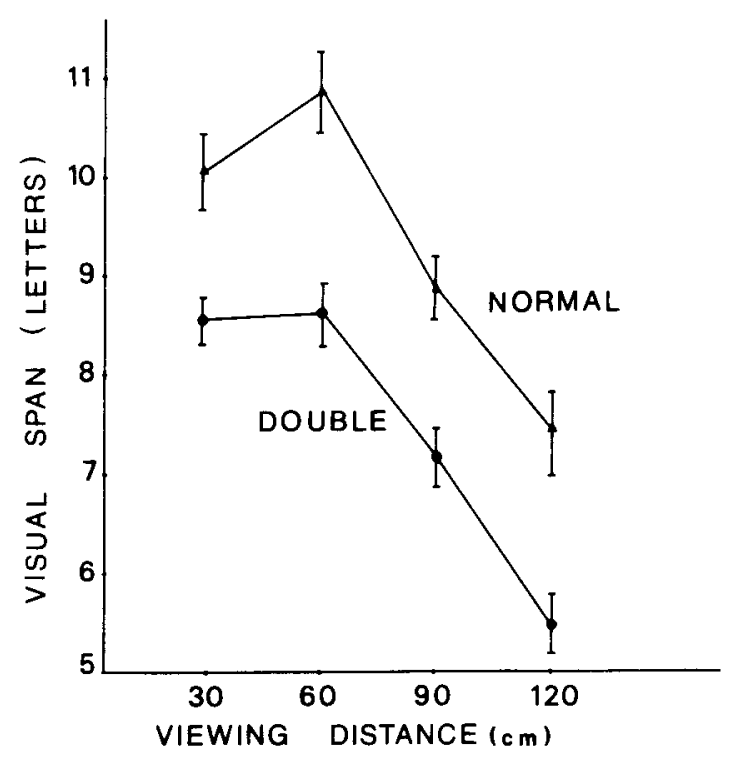

Figure 3. Visual span in the visibility experiment as a function of viewing distance. Span is defined as the number of letters visible on each side of the fixation point with a response probability of better than $50 \%$. It was obtained from Figure 2 by finding the eccentricities at which the probit ogives intersect the $50 \%$ level. The error bars represent the error of estimation of these intersections as calculated by the probit procedure. Two standard errors are shown on each side of the means. Span is measured in letters, not in character spaces. In the double-space condition, one letter takes two character spaces [cf. Figure 1, lines marked (b)]. really a "half-span," since it refers not to the sum but to the average of these zones.

Figure 3 shows that as distance is increased from beyond $60 \mathrm{~cm}$ to $120 \mathrm{~cm}$, visual span decreases by about 3.5 letters. In number of visible letters, span is larger for normal spacing than for double spacing by almost 2 letters at every viewing distance. Thus, even though masking is stronger in the normal-space condition, more letters in a string can be seen.

Earlier theoretical considerations (cf. O'Regan, 1983) had suggested that the curves in Figure 3 should have lain on straight lines with slight negative gradients, and not have had maxima around $60 \mathrm{~cm}$. However, more recent calculations taking into account trigonometrical corrections related to the larger angular eccentricities occurring for small viewing distances, and taking into account the curvature of the screen, show that the curves are, in fact, highly consistent with predictions. Of course, the precise shape of the curves is of no consequence here, since the curves are simply being used as a reference for the reading experiment which follows.

It is important to note that the visual span measured here cannot be taken to give the true absolute value of the visual span in reading, but only an indication of its relative variation with changes in distance and spacing. This is because (1) use of a reduced alphabet of 10 instead of 26 letters will have improved visibility over its true value in reading; (2) the task involved reporting two letters, which may have caused a slightly greater processing load and so given rise to a smaller span than if only one letter had to be reported; and (3) the span was defined here in terms of an arbitrary $50 \%$ threshold, and choosing a different threshold would have given similar, although vertically displaced, curves.

\section{READING EXPERIMENT}

The visibility experiment provided parametric measures of visual span under various conditions of viewing distance and spacing. Under the simple hypothesis that, in reading, information intake rate must be matched to processing rate, saccade sizes and fixation durations should adjust to changes in visual span. The present experiment investigated these adjustments.

\section{Method}

As in the visibility experiment, four viewing distances $(30,60$, 90 , and $120 \mathrm{~cm}$ ) and two spacing conditions (normal and double) were used. Subjects were required to read easy texts of 12 lines (24 lines in the double-space condition) while their eye movements were recorded. In the double-spaced texts, each letter was followed by a space of the same width as that normally occupied by a single letter. Spaces between words were also followed by an extra space:

THIS IS SINGLE SPACING

TH I S I S D O U B LE S P A C I N G 
Table 2

\begin{tabular}{|c|c|c|c|c|c|c|c|c|}
\hline \multirow{2}{*}{$\begin{array}{l}\text { Sub- } \\
\text { ject }\end{array}$} & \multicolumn{8}{|c|}{ Order of Presentation } \\
\hline & 1 & 2 & 3 & 4 & 5 & 6 & 7 & 8 \\
\hline 1 & A 30 & G 90 & B 60 & $\mathrm{~F} 120$ & D120 & H 60 & C 90 & E 30 \\
\hline 2 & G 60 & F 30 & $\mathrm{H} 120$ & $\overline{\mathrm{B} 90}$ & D 90 & $\overline{\mathrm{A} 120}$ & C 30 & $\overline{\mathrm{E} 60}$ \\
\hline 3 & $\overline{\text { H } 90}$ & B 120 & $\overline{\mathrm{G} 30}$ & F 60 & $\overline{\mathrm{C} 60}$ & D 30 & $\overline{\mathrm{E} 120}$ & $\mathrm{~A} 90$ \\
\hline 4 & $\mathrm{C} 120$ & $\mathrm{D} 60$ & E 90 & $\overline{\mathrm{H30}}$ & B 30 & $\overline{F 90}$ & A 60 & $\overline{\mathrm{G} 120}$ \\
\hline
\end{tabular}

Note-A-H are the eight texts. The numbers are the four viewing distances. Underlined $=$ double spaced; not underlined $=$ nor . mally spaced.

There were eight texts, which could occur at each of the four distances and be printed with normally spaced or double-spaced characters. Four subjects were used. It would have been most satisfactory to use the same subjects as in the visibility experiment, but this was not possible. Only one subject was common to both experiments.

The experimental design used here was in most ways similar to that used in the visibility experiment: Two Latin squares (4 subjects $\times 4$ distances), one for normally spaced and one for doublespaced texts, were interleaved so as to give an alternating order of presentation of normal- and double-space conditions. For each subject, the order of distances for one spacing condition was the opposite of what it was for the other spacing condition. This was arranged in such a way that, within the presentation order, distances never increased or decreased twice consecutively. The eight texts were assigned to the conditions such that across the four subjects, each of the eight texts occurred twice in each of the two spacing conditions, and each text was presented once at each of the viewing distances. The design is given in Table 2.

Texts appeared line by line on the same ADM 3A terminal as in the visibility experiment. When the subject's eyes made a return sweep, this triggered the disappearance of the line of text just read and the appearance of the next line. This method of text presentation was used because, with the recording apparatus used, records are much better without the interference of vertical eye movements. Because lines were replaced instantaneously by the computer, the situation was very similar to ordinary reading. The subjects adapted to it easily.

Eye movements were recorded using a photoelectric scleral reflection technique. The subject sat in a dental chair with his head constrained by lateral supports. The computer sampled eye position every 5 msec. Reading of each text was preceded by a calibration phase, and during the reading of the text, the computer continuously corrected the calibration for slow shifts caused by head movements or other artifacts. This correction was done by assuming that each return sweep started from near the end of each line and brought the eye to a fixed position at the beginning of the next line. Accuracy of estimation of the exact position fixated by the eye in the line cannot be obtained in this way, but relative accuracy, in particular the size of the saccades made, was guaranteed to be the same across subjects and conditions and of the order of 1 character space. The automatic recalibration corrupted the recordings at points near line beginnings and line endings, so data for such points were not included in the analysis.

Saccade sizes and fixation durations were analyzed by computer, using an algorithm in which a saccade was defined as a change in eye position of more than one character space, taking less than $50 \mathrm{msec}$ and giving rise to a fixation lasting more than $50 \mathrm{msec}$.

\section{Results}

Figure 4 gives mean progression and regression sizes measured in number of letters skipped, as a function of reading distance. The data for regressions is given for interest only, as none of the effects are significant because of lack of data: regressions represented only $11 \%$ of all eye movements.

As supported by the figure, the data for progressions shows that these are significantly shorter (when measured in letters skipped) for double-spaced texts $[F(1,3)=168.3, p<.0001]$. There is no significant effect of viewing distance $[F(3,9)=2.49, p>.10]$. However, the existence of an interaction between distance and spacing $[F(3,9)=4.41, \mathrm{p}<.05]$ shows that there may be a difference in the way reading distance affects saccade size in the normal- and doublespace conditions. This is supported by Figure 4, which shows that, although overall there is a negligible effect of viewing distance, there may be a slight effect for the normal condition. The (nonsignificant) data for regressions in the normal condition go in the same direction.

Figure 5 gives mean fixation durations as a function of reading distance. Fixation durations increase systematically with viewing distance $[F(3,9)=5.14$, $\mathrm{p}<.05]$. The effect of letter spacing is marginally significant $[F(1,3)=6.06, p<.10]$, and the interaction of viewing distance with spacing is not significant $[F(3,9)=1.60]$.

\section{DISCUSSION}

\section{Saccade Sizes}

The reading experiment provides data on the variation of eye-movement behavior with reading distance and character spacing. Can these variations be explained in terms of the changes in visual span caused by the reading-distance and character-spacing manipulations? Under the hypothesis that, in reading, the eye moves to a point determined by the size of the zone of visibility, saccade sizes should change exactly in step with changes in visual span: the data in Fig-

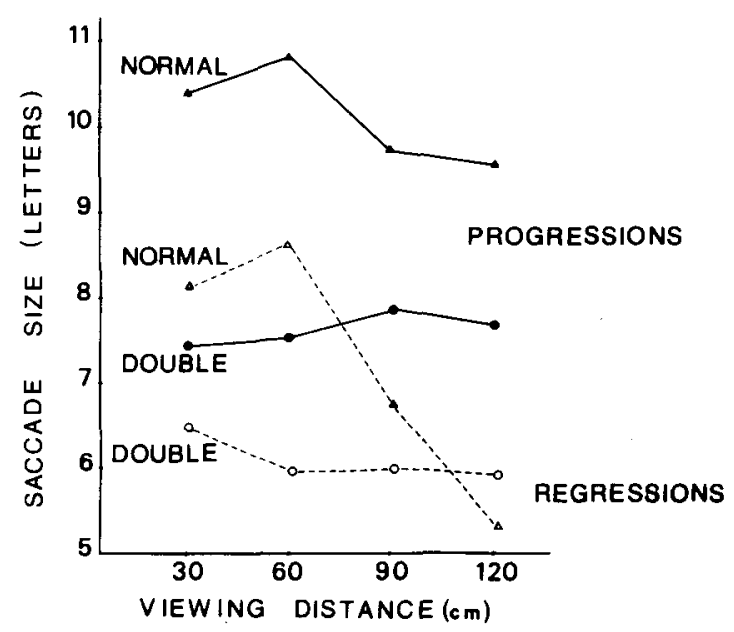

Figure 4. Mean saccade sizes, in number of letters skipped, in the reading experiment as a function of viewing distance. 


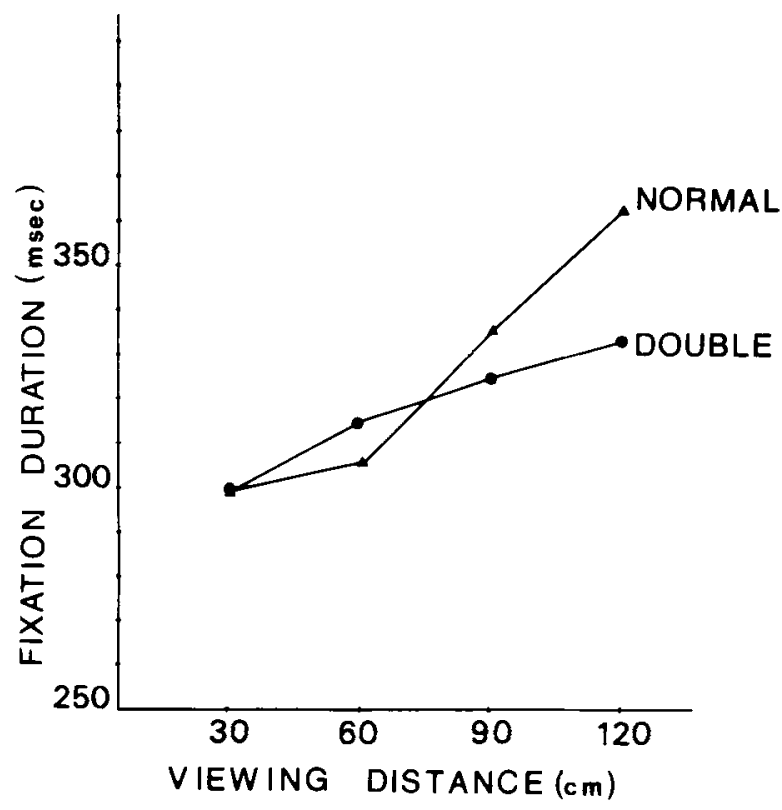

Figure 5. Mean fixation durations in the reading experiment as a function of viewing distance.

ure 4 should follow curves of the same shape as the visibility data in Figure 3. This is clearly not the case. The effect of reading distance in the reading experiment is much weaker than in the visibility experiment, particularly for double-spaced texts, for which there is no effect at all. Interestingly, however, the effect of character spacing is about the same in the two experiments: changing from single- to double-spaced characters changes visual span in the visibility experiment by about 2 letters. Saccade sizes in the reading experiment make a change of 2 to 3 letters when character spacing is changed from single to double.

The weakness or absence of a distance effect can be explained by supposing that saccade sizes in the reading experiment are controlled not by visual span, but by a distance-independent process. Candidates would be saccade-size-control strategies based on word length or linguistic structure, both of which are known to strongly influence saccade sizes (cf. Holmes \& O'Regan, 1981; Just \& Carpenter, 1978; O'Regan, 1980; Pollatsek \& Rayner, 1982).

However, the strong presence of a characterspacing effect cannot be accounted for in these terms. The fact that the effect is of about the same size as that found in the visibility experiment at first suggests that it is caused by the change in visual span. But this cannot be the case, since the visibility experiment shows that an analogous change in visual span can also be obtained by changing viewing distance from 60 to $120 \mathrm{~cm}$, and yet this had no effect in the reading experiment. A further factor must be postulated.

It is useful to take into account an observation made by the subjects in the reading experiment: In the double-space condition it was difficult to see where words began and ended. This is because, for the double-spaced texts, the ratio of interword to interletter spacing was much less favorable. This difference between normal and double-spaced texts may have been the cause for the character-spacing effect on saccade sizes. In the double-spaced texts, the subjects may have been forced to adopt a more "careful" eye-movement strategy in which they advanced by small constant steps in an effort to isolate word boundaries.

Thus, it appears that, in the visibility conditions used here, visual span was so large that it was not the limiting factor in determining saccade sizes. Other factors, possibly linguistic processing or eyemovement strategies related to word length and word boundary isolation, appear to have produced stronger constraints. An exception to this may be the normal-space condition, in which word boundaries were easy to see. Here a slight distance effect may have been present, indicating that visual span may have started to be a limiting factor.

Two other studies of the effect of viewing distance on eye-movement parameters are consistent with the present findings in that they also showed negligible variation of saccade size (measured in letters) with viewing distance (Morrison \& Rayner, 1981; O'Regan, 1983; see also Morrison, 1983). However, these two studies explained the lack of effect in terms of the tradeoff between retinal size and retinal acuity at differing eccentricities (cf. Javal, 1905; O'Regan, $1980,1983)$. The results of the visibility experiment clearly show that this tradeoff is not perfect, and so under the simple hypothesis that saccade sizes reflect visual span, saccade sizes in reading should in fact not be completely invariant with viewing distance changes. The fact that saccade sizes are largely invariant with viewing distance changes must therefore be attributed to the fact that other effects impose greater constraints on saccade size than does visual span.

\section{Fixation Durations}

The most obvious prediction that could be made about fixation durations would be to say that they should be proportional to the amount of information processed at each fixation. It seems reasonable to suppose that saccade sizes constitute a good estimate of the number of letters processed at each fixation. Since Figure 4 shows saccade sizes to be 2 letters larger in the normal-space condition, we would expect about 2 more letters to be processed per fixation in this condition and the average durations to be slightly longer. Figure 5 suggests that this is the case, although the effect was only slight $[F(1,3)=6.06$, $\mathrm{p}<.1$. A problem with this argument is that it predicts that the fixation durations should not depend on viewing distance, since saccade sizes did not. 
To deal with this problem, an additional factor can be invoked. Suppose that ease of sensory processing depends on how close to the limit of acuity are the letters to be identified. When letters are viewed from a long distance, their retinal size is small and only a small proportion of their distinctive features are visible: fixation durations should be long. When letters are viewed from a short distance, their retinal size is large and most of the distinctive features will be visible: fixation durations should be short. This hypothesis is compatible with the results, since Figure 5 shows that fixation durations do, in fact, increase with viewing distance. The fact that there is a slight suggestion that the normal-space condition has a steeper slope may be related to increased lateral masking. As shown by Jacobs (1979), this would cause an effective decrease in acuity and so a stronger dependence on viewing distance in the normal-space condition.

To summarize, it appears that two factors are involved in determining fixation durations. First, the number of letters being processed, as measured by saccade sizes, may influence fixation duration. However, this factor appears to play a fairly weak role: a change of 2 letters in the number of letters processed, as measured by saccade size, creates only about a 10msec difference in fixation duration. A second factor, which we suppose is related to the closeness of the letters being read to the limit of acuity, plays a stronger role. In going from 30 to $120 \mathrm{~cm}$, an increase of between 30 and $60 \mathrm{msec}$ occurs in fixation durations.

\section{CONCLUSION}

It was suggested in the introduction that, since visibility affects the very first stages of information intake in reading, changes in visibility should strongly affect the way the eye scans a text. The present experiments manipulated visibility by changing viewing distance and character spacing. This gave rise to changes of several letters in the "visual span," that is, in the number of letters clearly visible on each side of the fixation point.

The experiments showed that under visibility changes of this kind, saccade sizes are not primarily determined by visual span, but by other factors. These are possibly related to linguistic processing or to saccade control strategies affected by word length and the visibility of word boundaries. Visual span itself may become the limiting factor in determining saccade size in conditions in which interword spacing allows easy word isolation, when linguistic factors are not constraining, or when visibility is made substantially poorer than in the present experiments.

Fixation durations are highly sensitive to visibility changes. It seems, however, that the appropriate measure of visibility is not visual span, but nearness of individual letters to the reader's acuity threshold. As the size of the typography used approaches the limits of the reader's acuity, his fixation durations increase. There may be a relation of fixation duration to the strength of lateral masking or to the size of the zone processed at each fixation. The present experiments, however, did not sufficiently vary these parameters for an effect to be found, and the effect of nearness to the acuity threshold predominated.

\section{REFERENCES}

Bouma, H. Visual interference in the parafoveal recognition of initial and final letters of words. Vision Research, 1973, 13, 767-782.

Finney, D. J. Probit analysis. Cambridge: University Press, 1947.

Hackman, R. B., \& Tinker, M. A. Effect of variations in color of print and background upon eye movements. American Journal of Optometry, 1947, 4, 354-359.

Holmes, V. M., \& O'RegaN, J. K. Eye fixation patterns during the reading of relative-clause sentences. Journal of Verbal Learning and Verbal Behavior, 1981, 20, 417-430.

JAcoBs, R. J. Visual resolution and contour interaction in the fovea and periphery. Vision Research, 1979, 19, 1187-1196.

Javal, E. Physiologie de la lecture et de l'écriture. Paris: Alcan, 1905.

Just, M. A., \& Carpenter, P. A. Inference processes during reading: Reflections from eye fixations. In J. W. Senders, D. F. Fisher, \& R. A. Monty (Eds.), Eye movements and the higher psychological functions. Hillsdale, N.J: Erlbaum, 1978.

McConkie, G. W. Eye movements and perception during reading. In K. Rayner (Ed.), Eye movements in reading: Perceptual and language processes. New York: Academic Press, 1983.

Morrison, R. E. Retinal image size and the perceptual span in reading. In K. Rayner (Ed.), Eye movements in reading: Perceptual and language processes. New York: Academic Press, 1983.

Morrison, R. E., \& Rayner, K. Saccade size in reading depends upon character spaces and not visual angle. Perception \& Psychophysics, 1981, 30, 395-396.

O'REGAN, J. K. Saccade size control in reading: Evidence for the linguistic control hypothesis. Perception \& Psychophysics, $1979,25,501-509$.

O'REGAN, J. K. The control of saccade size and fixation duration in reading: The limits of linguistic control. Perception \& Psychophysics, 1980, 28, 112-117.

O'REGAN, J. K. Elementary perceptual and eye movement control processes in reading. In $\mathrm{K}$. Rayner (Ed.), Eye movements in reading: Perceptual and language processes. New York: Academic Press, 1983.

Paterson, D. G., \& Tinker, M. A. The effect of typography upon the perceptual span in reading. American Journal of Psy. chology, 1947, 60, 388-396.

Pollatsek, A., \& RAYner, K. Eye movement control in reading: The role of word boundaries. Journal of Experimental Psychology: Human Perception \& Performance, 1982, 8, 817-833.

RAYNER, $K$. The perceptual span and eye movement control during reading. In $\mathrm{K}$. Rayner (Ed.), Eye movements in reading: Perceptual and language processes. New York: Academic Press, 1983.

Rayner, K., Weli, A. D., \& Pollatsek, A. Asymmetry of the effective visual field in reading. Perception \& Psychophysics, $1980,27,537-544$.

Tinker, M. A., \& Paterson, D. G. The effect of typographical variations upon eye movements in reading. Journal of Educational Research, 1955, 49, 171-184. 


\section{NOTES}

1. Scores for letters presented on the left and the right were pooled, since, first, the existence of such differences would be irrelevant to the present investigation, and second, these differences were small and went in different directions for the four subjects (total left/right error ratios were: $54 / 46,40 / 60,58 / 42$, and $57 / 43$ for the four subjects).
2. Rayner, Well, and Pollatsek (1980) claim that the perceptual span in reading is asymmetrical, being larger on the right than on the left of the point fixated.

(Manuscript received March 31, 1983; revision accepted for publication July 25, 1983.) 